\title{
Structure Improvement and Flow Field Analysis of Condenser in Freeze - drying Equipment
}

\author{
Runling Peng ${ }^{1, a^{*}}$, Shasha Yin ${ }^{1, b}$, Hao Zhang ${ }^{1, c}$, Wei Cao ${ }^{1, d}$ and Ning Wang ${ }^{1, e}$ \\ 'School of Mechatronic Engineering, Xi'an Technological University, 710021 Xi'an, PRC \\ apengrunling@163.com, b1934332121@qq.com, '986950430@qq.com, 'caowei1998@126.com, \\ e1050088605@qq.com
}

*The corresponding author

Keywords: Condenser; Freeze - dry; Finite element; Flow field

\begin{abstract}
The condenser of freeze-dryer is used to capture the water vapor from the drying chamber A common problem of the condenser is that the frost is non-uniform and prone to "ice plugs". This paper studies the condenser of large food freeze-drying equipment, the structure of the condenser shell is changed from cylindrical to cone-cylinder, and the arrangement of condensing pipes in the condenser is changed to the non-uniform, condensing pipes is relatively sparse near the inlet, and it is relatively dense close to the outlet. The flow field in the condenser was analyzed by the finite element analysis method. The simulated result indicated that the vapor in the cone-cylinder shell is easy to form vortex, the streamline is uniform and long, and prolong the residence time of water vapor in the condenser, which is conducive to the uniform frosting and increase water vapor condensation rate.
\end{abstract}

\section{Introduction}

Freeze-drying orks by freezing the material and then reducing the surrounding pressure to allow the frozen water in the material to sublimate directly from the solid phase to the gas phase 1]. Widely used in medicine, food, biological products and preparation of nanomaterial. However, whether it is domestic equipment or the import of the freeze drying equipment, the common drawback is that the water vapor is too concentrated at the entrance of the condenser, easy to cause "ice plugs", lead to the low efficiency in water catching 2]. The performance of condenser is good or bad, which has a direct impact on the freeze drying effect or production efficiency, so it is necessary improve condenser structure to make it the frosting evenly and improve the water catching rate[3-4]. Especially for high energy consumption large-scale food freeze drying equipment, it is more important to solve this problem 5-6]. In this paper the condenser of large-scale freeze-drying equipment was modified by Dexi Wang's condenser. In order to improve the frosting uniformity and water catching rate 7].

\section{Structure Design of Condenser}

The condenser is a low temperature condensate pump for pumping steam. 1 grams of water in the $133 \mathrm{~Pa}$ vacuum degree, the volume of close to $1000 \mathrm{~L}$. If a working vacuum degree is $133 \mathrm{~Pa}$ and the amount of sublimation is $60 \mathrm{Kg}$ per hour of the freeze drying machine, requirements of the vacuum system pumping capacity is equivalent to $6 \times 107 \mathrm{~L} / \mathrm{h}$, need to have 28 pumps which pumping speed is $600 \mathrm{~L} / \mathrm{S}$, this is virtually impossible to achieve[8]. Therefore, the pumping system on the freeze drying machine, in addition to the use of Steam-jet Pump, you must set the condenser in order to remove water vapor, to achieve the drying of the material. This study takes the shelf area is $100 \mathrm{~m}^{2}$ of the food freeze-drying equipment as an example to design the condenser. Condenser limit vacuum is $5 \mathrm{~Pa}$, no-load pumping air time is $0.5 \mathrm{~h}$, condensation pipes working temperature is $-50{ }^{\circ} \mathrm{C}$ [9-10], the cooling time is not more than $1 \mathrm{~h}$. Through to the domestic and foreign large and medium-sized freeze drying machine investigation results show that the condensing area of condenser is related to the shelf area of freeze-drying chamber. And the area ratio is $(3 \sim 4): 1$, So 
the design of the area of the condensing pipes is about $300 \mathrm{~m}^{2}$. In order to improve the uniformity of condenser frost and water catching efficiency, in this design the shape of the shell of the condenser is changed from the traditional cylindrical to the cone-cylinder shaped. The arrangement of condensing pipes in the condenser, from the uniform layout to the inlet of a sparse and outlet dense non-uniform layout, the specific structure shown in Fig. 1 [11-12].

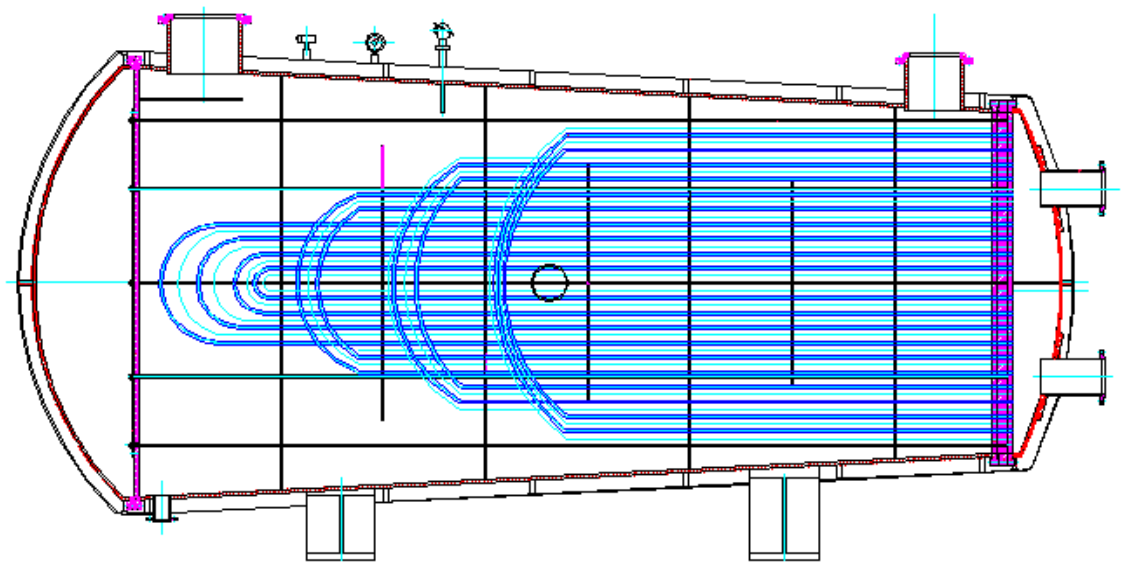

Figure 1. The structure of condenser

\section{Analysis of Inner Flow Field in Condenser}

In order to verify the structure of the condenser was improved is conducive to uniform frosting, first using Solid works to establish the three-dimensional model of the condenser, as shown in Fig. 2

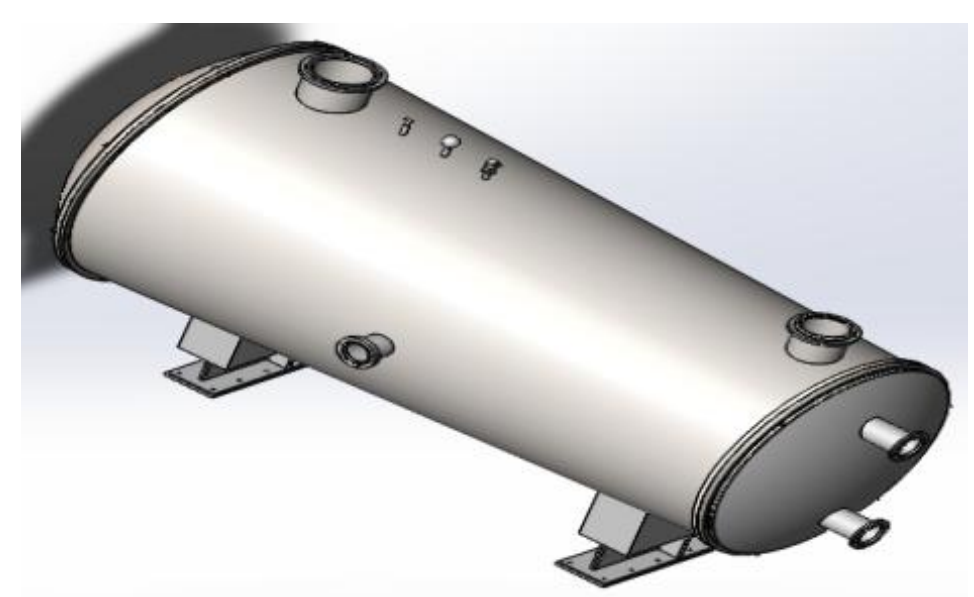

Figure 2. Three-dimensional model of condenser

ANSYS is used to analyze the flow field in the condenser. In the analysis of flow field, some simplified treatment was conducted on the structure of the condenser. Before the meshing, remove some insignificant parts (such as observation windows, pressure gauges, etc.), and only the analysis of the flow field inside the shell is carried out. Before the analysis of the flow field, the mesh cell size is 50mm, and the mesh shape using trihedron mesh, as shown in Fig. 3[13]. 


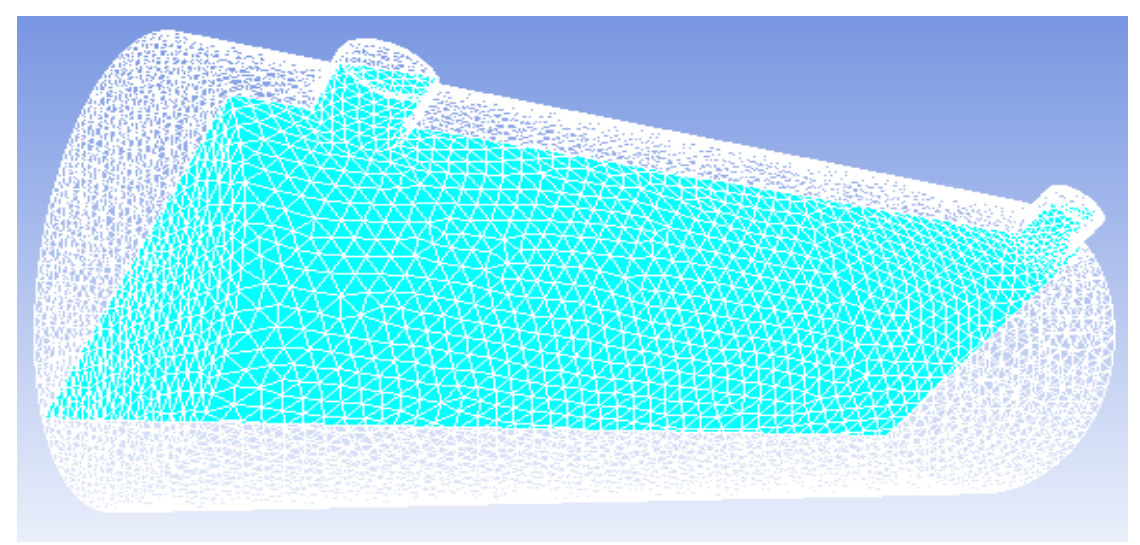

Figure 3. The mesh of condenser

Solving process: The turbulence model is selected as the fluid model, and the energy equation is chosen as the governing equation, and the fluid medium is the water vapor(assuming no consideration of the effects of permanent gases). In order to facilitate comparison, in the structure before and after improvement, using the same initial conditions and boundary conditions: The working pressure of the freeze dryer is $133 \mathrm{~Pa}$ and gas flow velocity on the outlet is $6.24 \mathrm{~m} / \mathrm{s}$ (the approximate calculation is based on pump pumping speed). The outlet temperature is set at $0{ }^{\circ} \mathrm{C}$. Because of the main content is the analysis of the flow field inside the shell is carried out, so the analysis of the results should be cut open its internal flow field diagram[14].

\section{Calculation Results and Analysis}

The velocity vector diagram of cylindrical condenser and cone-cylinder condenser is shown in Fig 4; s density distribution is shown in Fig. 5; and the flow Path-lines is shown in Fig. 6.

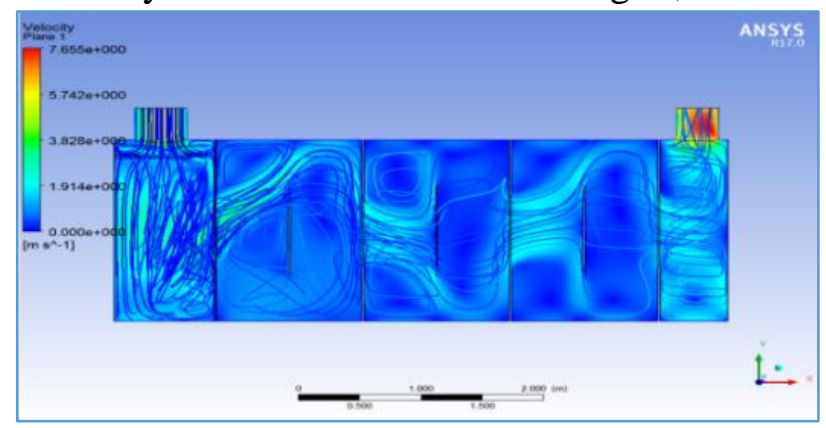

Figure a. Cylindrical condenser

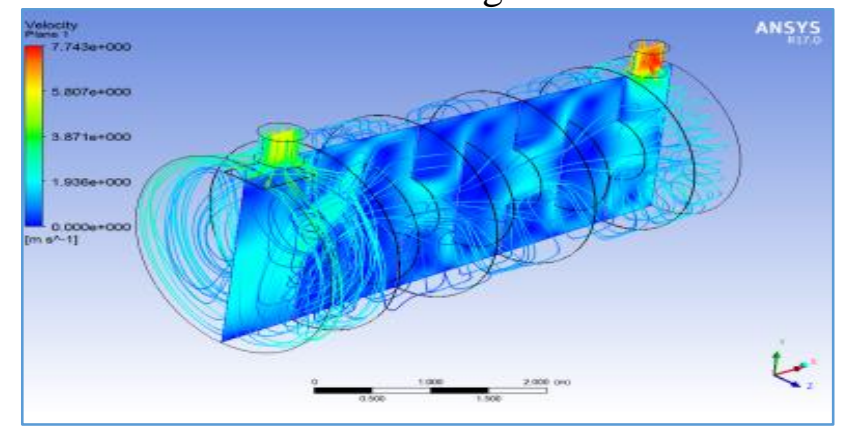

Figure b. Cone-cylinder condenser

Figure 4. Velocity vector diagram of condenser

It can be seen from the Fig. 4, the velocity of the cylindrical condenser is relatively uniform, although the velocity is larger when the flow through the baffle-plate, it is not obvious. While the cone-cylinder condenser, the gas flow through the baffle-plate, the gas velocity is very large, and the vortex is easy to form between the baffle-plate and the baffle-plate, which is conducive to the gas to stay in the condenser for a longer time, so as to improve the efficiency of frosting[15]. 


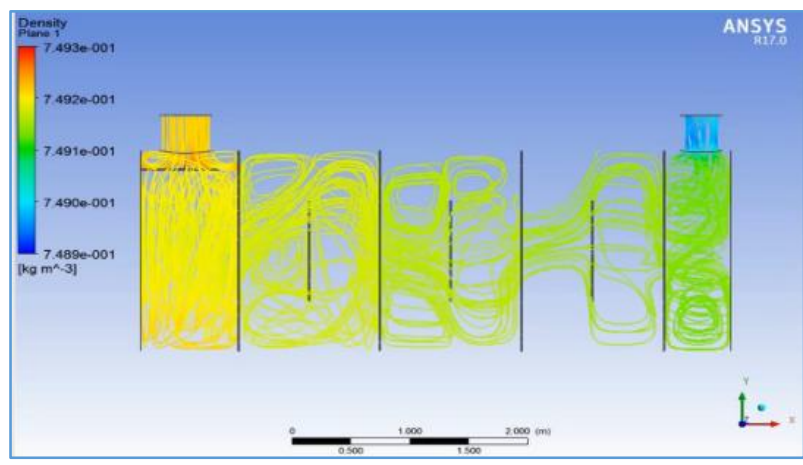

Figure a. Cylindrical condenser

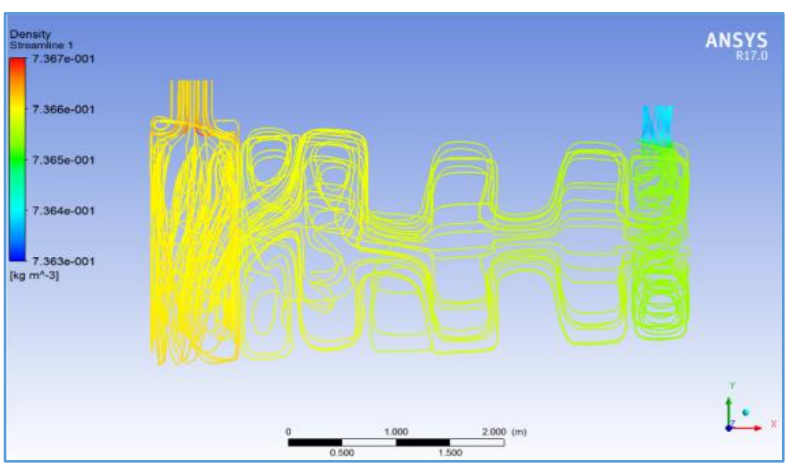

Figure b. Cone-cylinder condenser

Fig 5. Density distribution diagram in condenser

It can be seen from Fig. 5 that whether it is a cylindrical or Cone-cylinder condenser, the use of baffle-plate after the internal gas distribution are high inlet density, outlet density is low, and the difference is very small. If the use of cylindrical shell, the condensing pipes evenly arranged inside the condenser, it is bound to lead to the inlet to the frosting rate is high, the outlet frosting rate is low, resulting in uneven frost, easy to form the "ice plugs" phenomenon at the inlet. If the use of cone-cylinder shell, structure of the internal condensing pipes is inlet sparse and outlet dense, the structure shown in Fig. a, is conducive to the condensing pipes frost thickness tends to be the same at the inlet and outlet, to prevent ice plugs phenomenon.

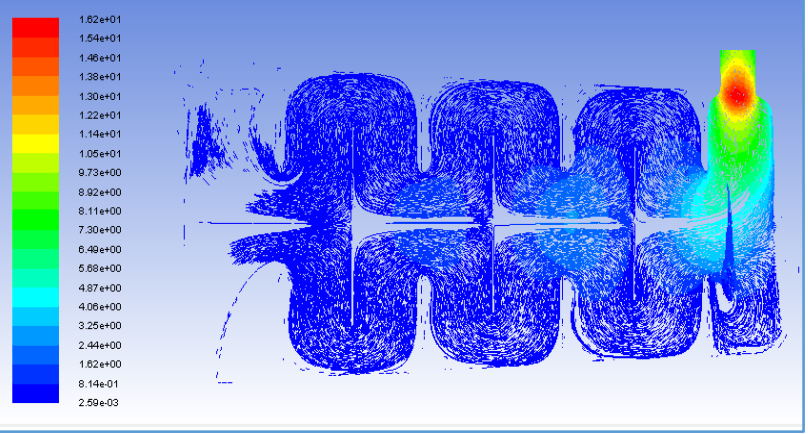

Figure a. cylindrical condenser

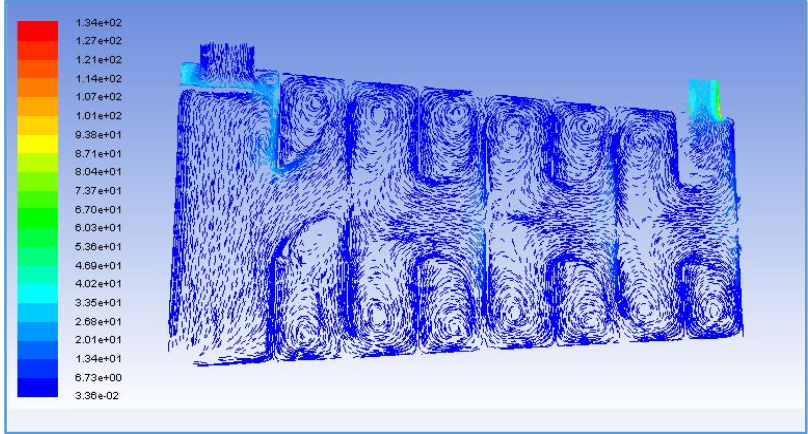

Figure b. Cone-cylinder condenser

Figure 6. The flow path-lines of condenser

As can be clearly seen from the gas flow path-lines in the condenser of Fig. 6. The gas in the Cone-cylinder condenser is easy to form a vortex at the baffle and the baffle plate, the gas travel is obviously increased, the time of Stay of the gas inside the shell is obviously lengthened, which is conducive the gas more fully contact with the condenser to improve the rate of frosting[16-17].

\section{Conclusion}

Through the above analysis, the structure of the condenser shell from the cylindrical to Cone-cylinder, condensing pipes layout from uniform distribution to the non-uniform,condensing pipes is relatively sparse near the inlet, and it is relatively dense close to the outlet. The baffle and baffle plate easy to form vortex, the gas travel is increased, prolong the residence time of water vapor in the condenser, so the frosting uniformity and the frosting rate of the condenser can be improved, and the "ice plugs" Phenomenon can be avoided effectively, which caused by the gas nearest the inlet was frozen on the cold surface, thereby enhancing the freeze-dryer performance of the catch water as well as work efficiency and quality of work. The analysis can provide a theoretical to design the condenser of large-scale freeze-drying equipment. However, the analysis does not consider the phase change heat transfer and frost growth on the condensing pipes in the condenser, future studies can consider the effects of these factors to simulate more accurate and real frosting situation. 


\section{Acknowledgements}

The Project Supported by Natural Science Basic Research Plan in Shaanxi Province of China (Program No. 2016JM5083);

Scientific Research Program Funded by Shaanxi Provincial Education Department (Program No.15JK1334).

\section{References}

[1] C.C. Cheng, S.M. Tsai and H.P. Cheng: International Communications in Heat \& Mass Transfer, Vol. 58 (2014) No.58, p.111.

[2] X.Q. Li: Journal of Ourval of longyan Univfrsity, Vol.6(2007)No.225,p.87.(In Chinese)

[3] Fettaka S, Thibault J and Gupta Y: International Journal of Heat \& Mass Transfer, Vol. 1 (2013) No.60, p.343.

[4] Stewart S W, Shelton S V: Applied Thermal Engineering, Vol. 14--15 (2010) No.30, p.2096

[5] X.Y. Zhang, X.L.Yuan and D. Hunag: Fluid Machinery, Vol.12(2005)No.32,p.305.(In Chinese)

[6] C.H. Xu, L. Hao and F.C. Xiong: Vacuum\&Cryogenics, Vol.3(2002)No.8,p.162.(In Chinese)

[7] Yao airu, Sama Ryoji: Design and experimental study on water vapor condenser of large scale food freeze drying machine.Kyowa Vacuum Engineering, Ltd. (1-18-17, Nishi-shim bashi , Minato-Ku, 105)

[8] F.F. Zhang, H.D. Han: Vacuum\&Cryogenics, Vol.1(2007)No.13,p.52.(In Chinese)

[9] aseli Y, Dincer I and Naterer G F: International Journal of Heat \& Mass Transfer, Vol. 9 (2008) No.51, p. 2462.

[10] Y. Liu, J. Shen, T.H. Zou: Vacuum\&Cryogenics, Vol.4(2004)No.10,p.230.(In Chinese)

[11] Salimpour M R: International Communications in Heat \& Mass Transfer, Vol. 9 (2008) No.35, p.1190.

[12] Jamshidi N, Farhadi M and Ganji D D: Applied Thermal Engineering, Vol. 1 (2013) No.51, p.644.

[13] Sanaye S, Hajabdollahi H: Applied Thermal Engineering, Vol. 14 (2010) No.30, p.1937.

[14] Parring A, Saarne A: International Journal of Refrigeration, Vol. 4 (2011) No.34, p.1066.

[15] Prabhanjan D G, Raghavan G S V and Rennie T J: International Communications in Heat \& Mass Transfer, Vol. 2 (202) No.29, p.185.

[16] Y.P. Chen, Y.J. Sheng and C.l. Dong: Applied Thermal Engineering, Vol. 1 (2013) No.50, p.1035.

[17] Sv P, Vs P, Db S: Journal of Fluid Mechanics, Vol. 62 (1974) No.62, p.117. 